\section{Granulomatous hypophysitis by Mycobacterium gordonae in a non HIV-infected patient}

\author{
Juan José Padilla-Martínez,' \\ Salvador González-Cornejo, ${ }^{1}$ \\ Lucía Elizabeth Álvarez-Palazuelos,' \\ Jesús Alejandro Villagómez-Méndez, \\ Erwin Chiquete, ${ }^{2}$ \\ José Alfredo Domínguez-Rosales, ${ }^{3,4}$ \\ Ismael Espejo-Plascencia, ${ }^{3}$ \\ Esteban González-Díaz, ${ }^{5}$ \\ José Rodrigo Torres-Baranda, ${ }^{6}$ \\ José Luis Ruiz-Sandoval, ${ }^{1,7}$
}

'Department of Neurology and

Neurosurgery; ${ }^{2}$ Department of Internal

Medicine; ${ }^{3}$ Department of Pathology;

${ }^{4}$ Department of Molecular Biology;

${ }^{5}$ Department of Infectious Diseases, from

the Hospital Civil de Guadalajara "Fray

Antonio Alcalde"; 'Department of

Molecular Biology and Genomics;

${ }^{7}$ Department of Neurosciences, from the

Centro Universitario de Ciencias de la

Salud (CUCS), Universidad de

Guadalajara, Jalisco, México

\section{Abstract}

Lymphocytic or granulomatous hypophysitis is a rare entity with a difficult diagnosis. Our objective was to report a patient with nontuberculous granulomatous hypophysitis. An HIV-negative 45-year old man with confusional state, subacute ophthalmoplegia, and clinical and laboratory findings of panhypopituitarism was seen in the emergency unit. A cranial MRI showed a sellar mass suggestive of hypophysitis. After an unsuccessful attempt with steroids and antituberculous drugs the patient died. Post-mortem histopathology revealed granulomatous lesions and restriction fragment length polymorphism analysis confirmed the presence of Mycobacterium gordonae's DNA. In conclusion, we should consider granulomatous hypophysitis in the differential diagnosis of non-secreting hypophyseal tumors. The etiology of a pituitary granuloma by a non-tuberculous mycobacteria is best reached by histopathological techniques and molecular assays. The optimal therapy is yet to be established.

\section{Introduction}

The pituitary region is susceptible to involvement by cystic, neoplastic, infectious and inflammatory processes. ${ }^{1}$ Granulomatous hypophysitis $(\mathrm{GH})$ is an inflammatory disorder characterized by the formation of granulomas frequently associated with tuberculosis, sarcoidosis, syphilis, and lymphocytic adenohypophysitis. This entity usually presents with systemic symptoms such as high fever and hormonal disturbances. ${ }^{2}$

We describe a post-mortem case of granulomatous hypophysitis secondary to infection caused by Mycobacterium gordonae. To our knowledge, only two other cases of $\mathrm{GH}$ caused by non-tuberculous mycobacteria infection (Mycobacterium malmoense and Mycobacterium tokaiense) in non-compromised hosts have been reported to date. ${ }^{3,4}$

\section{Case Report}

A 45-year-old man presented with a sixmonth history of weight loss, anorexia, vomiting, malaise and apathy. In the last month his condition worsened and headache, diplopia and left ptosis appeared. Neurological examination showed a person with slow mental processing, slow speech, affective flattening and left ophthalmoplegia (partial III cranial nerve palsy). No visual field disturbances, papilledema or meningeal signs were observed. General physical examination was unremarkable. Laboratory analyses only showed a low sodium blood level (114 mmol/L). A chest x-ray and a head CT scan were inconclusive and cerebrospinal fluid (CSF) was normal. After six days of hospitalization, fever, diarrhea and stupor appeared. A cranial MRI showed a sellar and parasellar heterogeneous mass, which in T1-weighted phase revealed a lesion with hypointense areas. In a T2-weighted phase this lesion was predominantly hyperintense with a hypointense center. After gadolinium administration, the lesion appeared heterogeneous with a parasellar extension toward the left cavernous sinus (Figure 1).

The measurement of plasma hypophysis
Correspondence: José Luis Ruiz Sandoval, Servicio de Neurología y Neurocirugía, Hospital Civil de Guadalajara "Fray Antonio Alcalde". Hospital 278, Guadalajara, Jalisco, México. CP: 44280. E-mail: jorulej-1nj@prodigy.net.mx

Key words: granuloma, hypophysis, non-tuberculous Mycobacteria, panhypopitituarism, pituitary gland.

Contributions: all authors have substantially contributed to the conception and design of the work and data analysis, take responsibility for the final version of the manuscript aapproved it for publication.

Conflict of interest: authors of this paper declare that the paper is original and has not been published or submitted for publication elsewhere and that there is no affiliation with any organization with a direct or indirect financial interest in the subject matter discussed in the manuscript that may affect the reporting of the work submitted.

Received for publication: 10 October 2009. Accepted for publication: 19 October 2009.

This work is licensed under a Creative Commons Attribution 3.0 License (by-nc 3.0).

(C)Copyright J.J. Padilla-Martínez et al., 2009 Licensee PAGEPress, Italy

Neurology International 2009; 1:e18

doi:10.4081/ni.2009.e18

hormones revealed a panhypopituitarism state. Based on the neuroimaging and hormonal findings, a presumptive diagnosis of hypophysitis was made. The patient was treated with steroid replacement, as well as with first- and second-line antituberculous drugs. Other laboratory studies were unremarkable, including serological tests for B and C hepatitis viruses, HIV, VDRL and Brucella, as well as erythrocyte sedimentation rate, C-reactive protein, antinuclear antibodies and rheumatoid factor.

Despite management, the patient died on
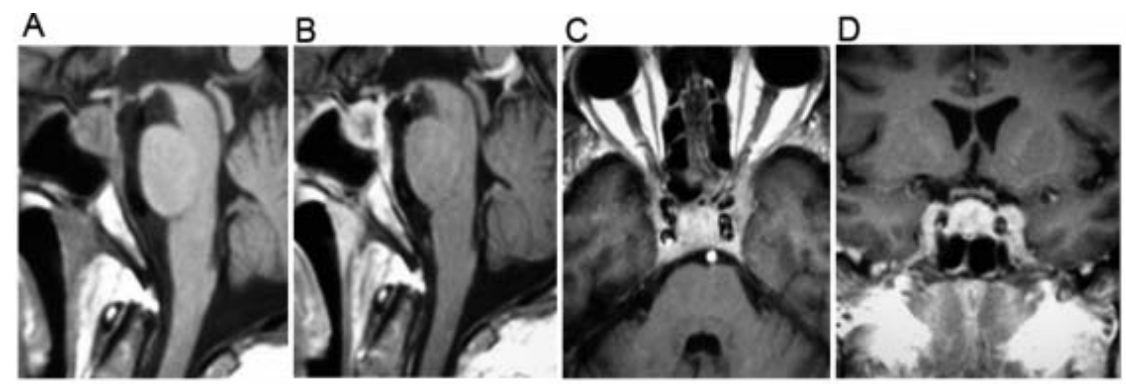

Figure 1. A cranial magnetic resonance imaging showed an intrasellar mass. (A) A sagittal T1-weighted image revealed a sellar lesion with hypointense areas. (B) A gadoliniumenhanced sagittal image showed an enhancing lesion with a hypointense center. Axial (C) and coronal (D) images demonstrated parasellar extension toward the left cavernous sinus. 
day 11 of hospitilization. The autopsy showed an enlarged, fibrous and thickened pituitary gland. Microscopically, a granulomatous cellular reaction suggestive of mycobacterial disease was observed; however, Ziehl-Neelsen, modified Kinyoun and Auramine-Rodamine staining of the specimen did not identify any acid-fast bacteria (AFB). Molecular diagnostic testing for potential mycobacterial pathogens was then performed. Total DNA was extracted from the hypophysis specimen using a modification of the lysis method described by Sritharan and Barker. $^{5}$ A PCR assay and restriction fragment length polymorphism (RFLP) analysis originally described by Roth $e t$ $a l$. with oligonucleotide primers of the 16S-23S spacer was conducted. ${ }^{6}$ DNA amplification experiment included positive controls (for $M$. tuberculosis H37Rv, M. chelonae, and $M$. kansasii) and a blank as well as negative controls. The amplified products were digested separately with 2 IU of the restriction enzyme HaeIII and CfoI, (Sigma, St. Louis, MO, USA), according to the manufacturer's recommendations. Fragment band sizes were estimated visually by comparison with appropriate controls (those positive for M. tuberculosis H37Rv, $M$. chelonae, and $M$. kansasii) in parallel with an extract of a whole hypophysis specimen and a 50 bp ladder (Fermentas, Hanover, USA). RFLP assay identified the genotypic characteristics of M. gordonae.

\section{Discussion}

Even after the HIV pandemics and the great use of immunosuppressive drugs, $\mathrm{GH}$ by nontuberculous mycobacteria remains an uncom-

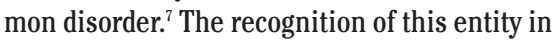
the differential diagnosis of abnormal sellar masses is important. In cases of $\mathrm{GH}$ it has been reported a disproportional degree of hormonal disturbances in relation to the size of the sellar mass. ${ }^{8}$ The most common manifestations are hypopituitarism, hyperprolactinemia, diabetes insipidus, visual field disturbances or aseptic meningitis. Nevertheless, none of these disorders is specific for this condition. ${ }^{1}$ Adequate diagnostic evaluation includes assessment of pituitary gland function, head magnetic resonance imaging and the investigation of the potential systemic disease. The trans-sphenoidal approach is the ideal method for diagnosis and local cure in masses suggestive of intrasellar granulomas, as it avoids cerebrospinal fluid contamination by infec- tious material. ${ }^{8}$ In our patient, the critical systemic and neurological condition made this surgical approach impossible.

The other ${ }^{2}$ reported cases of $\mathrm{GH}$ due to nontuberculous mycobacteria with no history of immunosupression included a 32-year-old woman who presented with fever, headaches, nausea, vomiting and a six-month history of diabetes insipidus and amenorrhea. ${ }^{3}$ In the second case, a 36 -year-old man presented with diabetes insipidus beginning three months prior, without other hormonal disturbances or any symptoms of mycobacterial meningitis. In both cases, Gd-MRI revealed an enhanced intrasellar mass of heterogeneous appearance with suprasellar extension and thickening of the pituitary stalk. ${ }^{4}$ In both patients, a mass lesion was completely removed and histological examination of the surgical specimen showed an epithelioid cell granuloma with caseous necrosis, but the acid-fast staining did not identify any AFB organisms. PCR and DNA sequencing to detect the non-tuberculous mycobacteria infection were used.

$M$. gordonae has been referred to as a tap water bacillus. It is also found in soil, whirlpools, and swimming pools. In healthy people it can be isolated from mucous membranes, urine, and gastric fluid. In the light of the widespread presence of this pathogen, infection with $M$. gordonae seems exclusively determined by host characteristics, and not by pathogen or exposure variables. $M$. gordonae has often been involved in pseudo-epidemics, where positive cultures were caused by contaminated tap water, fountains, ice machines, antimicrobial and laboratory solutions, aerosol devices and bronchoscopes. ${ }^{9-11}$ Despite this apparent vulnerability for our report, we believe this mycobacteria was responsible for this condition in view of all the clinical, radiological and histopathological test results.

The optimal therapy for patients with $\mathrm{GH}$ due to non-tuberculous mycobacteria is not defined in the literature. Although it is universally accepted that chemotherapy is essential for a successful treatment of intracranial granulomas, there is no consensus regarding the regimen of drugs or the duration of therapy. ${ }^{10}$ Antituberculous treatment must be opportunely initiated in patients who are thought to have GH since the mortality rate for mycobacterial central nervous system infection remains high. ${ }^{8}$

In conclusion, we should consider $\mathrm{GH}$ in the differential diagnosis of non-secreting hypophyseal tumors. The etiology of a pituitary granuloma by a non-tuberculous mycobacteria is best reached by histopathological techniques and molecular assays. The optimal therapy is yet to be established.

\section{References}

1. Lipscombe L, Asa SL, Ezzat S. Management of lesions of pituitary stalk and hipothalamus. Endocrinologist 2003;13:38-51.

2. Honegger J, Fahlbusch R, Bornemann A, et al. Lymphocytic and granulomatous hypophysitis: Experience with nine cases. Neurosurgery 1997;40:713-23.

3. Florakis D, Kontogeorgos G, Anapliotou M, et al. Isolated pituitary granuloma by atypical Mycobacterium in a nonimmunosuppressed woman. Clin Endocrinol 2002;56: 123-6.

4. Kondo A, Mori K, Iwata J, et al. Caseus necrotic granuloma in the pituitary stalk due to nontuberculous mycobacteria (Mycobacterium Tokaiense) infection. Neur Med Chir 2006;46:80-3.

5. Sritharan V, Barker RH. A simple method for diagnosis $M$. tuberculosis infection in clinical samples using PCR. Mol Cell Probes 1991;5:385-95.

6. Roth A, Reischl U, Streubel A, et al. Novel diagnostic algorithm for identification of mycobacteria using genus-specific amplification of the 16S-23S rRNA gene spacer and restriction endonucleases. J Clin Microbiol 2000;38:1094-104.

7. Cheung CC, Ezzat S, Smyth HS, Asa SL. The spectrum and significance of primary hypophysitis. J Clin Endocrinol Metab 2006;86:1048-53.

8. Sinha S, Singh A, Tatke M, Singh D. Hypophyseal tuberculoma: Direct radiosurgery is contraindicated for a lesion with a thickened pituitary stalk: Case report. Neurosurgery 2000;46:735-8.

9. Griffith DE, Aksamit T, Brown-Elliott BA, et al. An official ATS/IDSA statment: Diagnosis, treatment, and prevention of nontuberculous mycobacterial diseases. Am J Respir Crit Med 2007;175:367-410.

10. den Broeder AA, Vervoort G, van Assen S, et al. Disseminated Mycobacterium gordonae infection in a renal transplant recipient. Transpl Infect Dis 2003;5:151-5.

11. Arnow PM, Bakir M, Thompson K, Bova JL. Endemic contamination of clinical specimens by Mycobacterium gordonae. Clin Infect Dis 2000;31:472-6. 